\title{
FDA Inactivation
}

National Cancer Institute

\section{Source}

National Cancer Institute. FDA Inactivation. NCI Thesaurus. Code C162847.

FDA initiated and completed the inactivation of a registration or listing record due to inaccuracies, incompleteness or incompliance. 